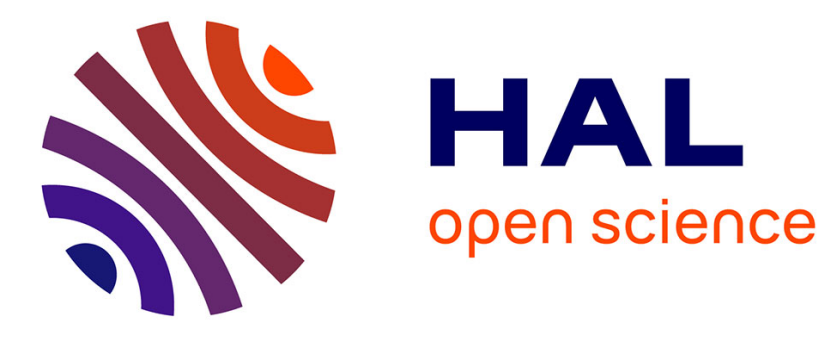

\title{
Les fluoronitrures de terres rares
}

Michel Pezat, Bernard Tanguy, Marcus Vlasse, Josik Portier, Paul

Hagenmuller

\section{To cite this version:}

Michel Pezat, Bernard Tanguy, Marcus Vlasse, Josik Portier, Paul Hagenmuller. Les fluoronitrures de terres rares. Journal of Solid State Chemistry, 1976, 18 (4), pp.381-390. 10.1016/0022-4596(76)901225. hal-03480278

\section{HAL Id: hal-03480278 https://hal.science/hal-03480278}

Submitted on 14 Dec 2021

HAL is a multi-disciplinary open access archive for the deposit and dissemination of scientific research documents, whether they are published or not. The documents may come from teaching and research institutions in France or abroad, or from public or private research centers.
L'archive ouverte pluridisciplinaire HAL, est destinée au dépôt et à la diffusion de documents scientifiques de niveau recherche, publiés ou non, émanant des établissements d'enseignement et de recherche français ou étrangers, des laboratoires publics ou privés. 


\title{
Les Fluoronitrures de Terres Rares
}

\author{
MICHEL PEZAT, BERNARD TANGUY, MARCUS VLASSE, \\ JOSIK PORTIER ET PAUL HAGENMULLER
}

Laboratoire de Chimie du Solide du C.N.R.S., Université de Bordeaux I, 35I, cours de la Libération, 33405, Talence, France

\begin{abstract}
The reaction of rare-earth nitrides with fluorides or the pyrolysis of these fluorides in gaseous ammonia atmosphere leads, in the case of lanthanum, cerium, and gadolinium, to nitride fluorides of general formula $L n \mathrm{~N}_{x} \mathrm{~F}_{3-3 x}$. They crystallize with a tluorite-type structure which can be described in a consistent manner in terms of a Willis model. The magnetic properties of $\mathrm{Gd}_{3} \mathrm{NF}_{6}$ have been studied.
\end{abstract}

Les fluoronitrures à caractère ionique préparés et étudiés à ce jour sont peu nombreux. Ce fait peut paraître surprenant dans la mesure où l'on considère cette famille comme dérivant des oxydes correspondant par remplacement de deux ions $\mathrm{O}^{2-}$ par le couple $\mathrm{N}^{3-}+\mathrm{F}^{-}(I)$. En fait ce mécanisme ne tient pas compte des différences profondes qui existent entre la liaison $M-\mathrm{N}$, covalente ou métallique, et la liaison $M-\mathrm{F}$ à caractère ioniquc. Celles-ci, ainsi que la difficulté de préparation et de manipulation des produits réactionnels, fluorures et nitrures, peuvent expliquer le petit nombre de résultats publiés dans ce domaine.

Lorsque nous nous sommes intéressés à ce typo de composés, sellls étaient connus les fluoronitrures de thorium et de zirconium préparés par Juza, Sievers et Jung $(2,3)$, celui d'uranium obtenu par Yoshihara, Kanno et Mukä̈bo (4), ainsi que les fluoronitrures de magnésium préparés par Andersson (5).

Depuis lors les fluoronitrures de calcium, de strontium et de baryum ont été mis en évidence simultanément par Ehrlich, Linz et Steifert (6) d'une part, Galy, Jaccou et Andersson (7) d'autre part. Marchand et Lang ont par ailleurs préparé les fluoronitrures de zinc (8). Enfin dans deux articles récents Jung et Juza complètent l'étude structurale des fluoronitrures de zirconium et d'uranium $(9,10)$.

Lors de publications antérieures nous avons décrit la préparation et quelques propriétés des fluoronitrures de lanthane et de gadolinium $(11,12)$. Il nous a paru tentant de compléter cette étude et de l'étendre au cérium, à l'europium et au thulium. Le choix de l'europium se justifiait d'une part pour ses propriétés optiques (intensité des transitions électroniques), d'autre part par l'existence de deux degrés d'oxydation +II et +III; enfin il était tentant de comparer sur le plan cristallographique les résultats obtenus dans le cas du lanthane à ceux correspondant à une terre rare de petite taille telle que le thulium

\section{Méthodes Expérimentales}

La synthèse des fluoronitrures implique l'obtention préalable des fluorures et des nitrures correspondants. Les fluorures $\mathrm{CeF}_{4}$ et $L n \mathrm{~F}_{3}(L n=\mathrm{La}, \mathrm{Eu}, \mathrm{Gd}$ et $\mathrm{Tm})$ sont préparés par fluoration des oxydes à $500^{\circ} \mathrm{C}$. $\mathrm{CeF}_{3}$ et $\mathrm{EuF}_{2}$ sont obtenus par réduction de $\mathrm{CeF}_{4}$ et $\mathrm{EuF}_{3}$ par l'hydrogène sec respectivement à $400^{\circ} \mathrm{C}$ et $1000^{\circ} \mathrm{C}$. Pour la préparation des nitrures nous utilisons la méthode mise au point par Anselin: action à basse 
température de l'azote sous pression sur le métal partiellement hydruré (13).

Les fluoronitrures s'obtiennent selon deux méthodes:

-Interaction nitrure-fluorure. Les deux composés sont alors mélangés sous azote sec et désoxygéné dans une boîte à gants. Placés dans un creuset de molybdène, les mélanges sont introduits dans une bombe en alumine et chauffés sous une pression d'azote de quelques bar.

- La thermoammoniolyse des fluorures. Ceux-ci sont placés dans une nacelle en nickel et introduits à contre-courant d'azote dans un réacteur étanche en acier inoxydable. Après dégazage ils sont chaufiés sous une pression. d'ammoniac de quelques bar.

Les échantillons subissent un traitement thermique final:

- soit une trempe de l'ordre de $1000^{\circ} \mathrm{C} / \mathrm{h}$.

- soit des recuits de 200 à $300 \mathrm{~h}$ à des températures comprises entre 1000 et $400^{\circ} \mathrm{C}$ suivis d'un refroidissement rapide.

\section{Résultats Obtenus}

Lęssystèmes $L n N-L n F_{3}(L n=L a, C e, E u, G d$, Tm)

Cas où $L n=L a, C e, G d$. L'analyse radiocristallographique après traitement thermique des livers échantillons étudiés montre pour chacun de ces éléments la formation d'une phase nouvelle de symétrie cubique. Elle correspond à un fluoronitrure que nous conviendrons de réprésenter par la formule générale: $\operatorname{Ln} \mathrm{N}_{x} \mathrm{~F}_{3-3 x}$.

Pour le lanthane et le cérium la réaction débute à $600^{\circ} \mathrm{C}$. A $900^{\circ} \mathrm{C}$ elle est complète au bout de quinze heures. Pour le gadolinium des températures plus élevées sont nécessaires: à $1100^{\circ} \mathrm{C}$ la réaction est encore très lente, il faut atteindre $1300^{\circ} \mathrm{C}$ pour qu'elle soit totale au bout d'une quinzaine d'heures.

Les fluoronitrures de lanthane et de cérium comportent de larges domaines d'existence. Après trempe ce domaine correspond à $0.33 \leqslant x \leqslant 0.50$. Après recuit de $300 \mathrm{~h}$ à diverses températures comprises entre $700^{\circ} \mathrm{C}$ et $400 \mathrm{C}$, il diminue légèrement et correspond alors à $0.33 \leqslant x \leqslant 0.47$ (Fig. 1).

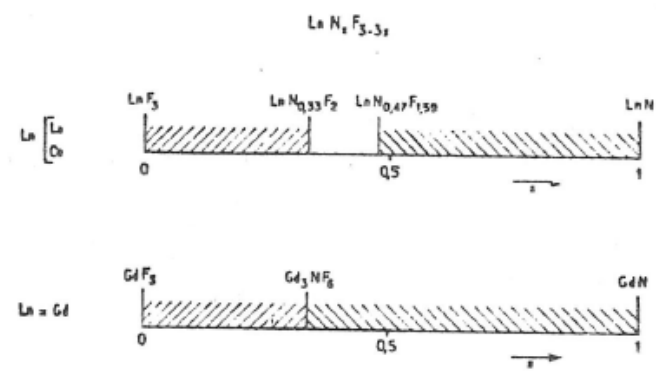

FIG. 1. Domaines d'existence des fluoronitrures de lanthane, de cérium et de gadolinium dans le cas d'échantillons recuits.

Dans le cas du gadolinium, quel que soit le traitement thermique, nous n'avons pu mettre en évidence de domaine d'existence.

La phase observée correspond à $x=0.33$, donc à la formule $\mathrm{Gd}_{3} \mathrm{NF}_{6}$ (Fig. 1). Du fait de la très grande réactivité de $\mathrm{GdN}$ vis-à-vis de l'oxygène et de la vapeur d'eau nous n'avons pu étudier le système $\mathrm{GdN}-\mathrm{GdF}_{3}$ à la chambre de diffraction $\mathrm{X}$ à haute température pour mettre en évidence un éventuel domaine d'existence.

L'analyse chimique confirme la composition des phases obtenues. Le tableau I rassemble les résultats obtenus pour les fluoronitrures de lanthane, de cćrium et de gadolinium correspondant à $x=0.33$.

Des mesures de susceptibilité magnétique effectuées entre 4.2 et $300 \mathrm{~K}$ ont permis de confirmer que le cérium était au degré d'oxydation +llI dans le fluoronitrure. L.a constante de Curie expérimentale $C=0.794$ est en bon accord avec la valeur théorique $C=0.819$ et exclut donc la présence de quantités notables de cérium +IV.

Les fuorcnitiures cie lanthane $e_{\iota}$ de gadolinium se présentent sous forme de poudres gris clair. Très stables thermiquement ils ne subissent aucune dissociation lorsqu'ils sont portés à $1000^{\circ} \mathrm{C}$ sous un vide de $10^{-6}$ torr.

La thermohydrolyse du fluoronitrure de lanthane ou de gadolinium à $900^{\circ} \mathrm{C}$ conduit à ia formation de l'oxyde correspondant. Pour $x=0.33$ le schéma réactionnel est le suivant:

$$
\begin{aligned}
6 \mathrm{LnN}_{0.33} \mathrm{~F}_{2}+ & 9 \mathrm{H}_{2} \mathrm{O} \rightarrow 3 \mathrm{Ln}_{2} \mathrm{O}_{3}+ \\
& +2 \mathrm{NH}_{4} \mathrm{~F}(\mathrm{~g})+10 \mathrm{HF}(\mathrm{g}) .
\end{aligned}
$$


Le fluoronitrure de cérium est obtenu sous forme d'une poudre de couleur vert clair. Contrairement aux fluoronitrures de lanthane ou de gadolinium il réagit instantanément avec l'oxygène de l'air. Il donne alors une poudre de couleur marron foncé. La très mauvaise définition des pics de diffraction $\mathrm{X}$ ne nous a pas permis d'identifier les phases en présence, qui sont évidemment mal cristallisées.

Cas de l'europium. L'étude du système EuN-EuF ${ }_{3}$ n'a pas permis de mettre en évidence la formation d'un fluoronitrure.

Jusqu' 4 i $400^{\circ} \mathrm{C}$ nitrure et fluorure ne réagissent pas. Au-dessus de cette température et sous pression d'azote d'un bar, la réduction de $\mathrm{Eu}^{3+}$ intervient suivant le schéma réactionnel:

$$
2 \mathrm{EuN}+4 \mathrm{EuF}_{3} \rightarrow 6 \mathrm{EuF}_{2}+\mathrm{N}_{2}(\mathrm{~g}) .
$$

Pour une pression de 15 bar nous n'avons pas observé de variation sensible du seuil de température de la réaction (2). Faute de pouvoir disposer d'azote sous plus forte pression dans l'état de pureté que nous imposait la réactivité de EuN vis-à-vis de l'eau et de l'oxygène, nous n'avons pu opérer à des pressions suffisamment élevées pour entraver la dissociation du nitrure EuN.

Pour des échantillons correspondant à un rapport molaire initial $\mathrm{EuF}_{3} / \mathrm{EuN}>2$ on observe la formation des phases $\mathrm{EuF}_{2+x}$ mises en évidence par Tanguy lors de l'étude du système $\operatorname{EuF}_{2}-\mathrm{EuF}_{3}(/ 4)$.

Pour un rapport $\mathrm{EuF}_{3} / \mathrm{EuN}<2$ le fluorure EuF $F_{2}$ produit par la réaction (2) coexiste au refroidissement avec le nitrure EuN en excès sans qu'il y ait réaction, même à $1377^{\circ} \mathrm{C}$, température de iusion de EuF 2 .

Faute de pouvoir isoler un fluoronitrure d'europium nous avons cherché à doper le fluoronitrure de lanthane par $\mathrm{Eu}^{3+}$. L'étude des propriétés optiques de l'europium dans cette matrice était en effet susceptible de nous apporter des informations sur la structure de $\operatorname{LaN}_{x} \mathrm{~F}_{3-3 x}$. En fait l'expérience nous a montré que lors des traitements thermiques de mélangus:

$$
(1+x) \mathrm{Laa}_{(0,0)} \mathrm{Eu}_{(0,0)} \cdot x \mathrm{LaN}
$$

ou:

$$
0.99 \mathrm{LaN}_{x} \mathrm{~F}_{3-3 x}-0.01 \mathrm{EuF}_{3}
$$

tels que $2.06 \leqslant$ (nombre d'anions)/(nombre de cations $) \leqslant 2.33$, la réduction de $\mathrm{Eu}^{3+}$ en $\mathrm{Eu}^{2+}$ intervenait également, conduisant à un mélange de fluoronitrure de lanthane et de $\mathrm{EuF}_{2}$.

Cas du thulium. Quel que soit le traitement thermique effectué, pour des températures allant jusqu'à la fusion du fluorure, à $1158^{\circ} \mathrm{C}$, nous n'avons pu observer la formation d'une phase ternaire entre $\mathrm{TmN}$ et $\mathrm{TmF}_{3}$.

\section{Le système $\mathrm{CeN}-\mathrm{CeF}_{4}-\mathrm{N}_{2}$}

Bien que nous nous soyons proposés initialement d'étudier les fluoronitrures correspondant au degré d'oxydation +III du cation, il était tentant d'essayer de préparer CeNF dont l'existence pouvait être envisagée par analogie avec le composé correspondant du thorium et de l'uranium $(2-4,9,10)$.

Dans ce but nous avons étudié le système $\mathrm{CeN}-\mathrm{CeF}_{4}$ sous pression d'azote de 15 bar, limite imposée par l'appareillage utilisé.

En fait l'expérience nous a montré que dans ces conditions, seule intervenait dès $400^{\circ} \mathrm{C}$ la réaction:

$$
2 \mathrm{CeN}+6 \mathrm{CeF}_{4} \rightarrow 8 \mathrm{CeF}_{3}+\mathrm{N}_{2}(\mathrm{~g}) .
$$

Pour des échantillons de rapport molaire $\mathrm{CeF}_{4} / \mathrm{CeN}$ inférieur à 3 le nitrure en excès réagit avec $\mathrm{CeF}_{3}$ formé lors de la réaction (3) pour donner le fluoronitrure trivalent.

Thermoammoniolyse des fuorures de terres rares

Cas de $\mathrm{LaF}_{3}, \mathrm{CeF}_{3}, \mathrm{GdF}_{3}$. L'expérierce a montré que l'ammoniac sous pression d'un bar réagissait sur $\mathrm{LaF}_{3}$ et $\mathrm{CeF}_{3}$ dès $900^{\circ} \mathrm{C}$ et sur $\mathrm{GdF}_{3}$ à partir de $1050^{\circ} \mathrm{C}$. Cette réaction se manifeste par un aépart de rluorure d'amtmonium qui se condense dans les parties froides de l'appareil. L'analyse radiocristallographique montre dans chaque cas la formation d'une phase cubique identique à celle mise en évidence lors de l'étude des systèmes $L n \mathrm{~N}-L n \mathrm{~F}_{3} \quad(L n=\mathrm{La}, \mathrm{Ce}, \mathrm{Gd})$. L'analyse chimique confirme l'identité de ces phases.

Dans le cas du lanthanc et du cérium on observe apres trempe le méme domaine d'existence pour les fluoronitrures correspondants $(0.33 \leqslant x \leqslant 0.50)$; de même pour 
le gadolinium, seule la phase correspondant à la composition $\mathrm{Gd}_{3} \mathrm{NF}_{6}$ a pu être isolée. La réaction s'écrit:

$L n \mathrm{~F}_{3}+4 x \mathrm{NH}_{3} \rightarrow L n \mathrm{~N}_{x} \mathrm{~F}_{3-3 x}+3 x \mathrm{NH}_{4} \mathrm{~F}(\mathrm{~g})$

avec

$$
\begin{cases}L n=\mathrm{La}, \mathrm{Ce} & 0.33 \leqslant x \leqslant 0.50 \\ L n=\mathrm{Gd} & x=0.33\end{cases}
$$

Cas de $E u F_{3}$. L'action de l'ammoniac sur $\mathrm{EuF}_{3}$ ne conduit pas à un fluoronitrure. Dès $600^{\circ} \mathrm{C}$ sous pression de 1 bar la réduction de $\mathrm{EuF}_{3}$ en $\mathrm{EuF}_{2}$ intervient:

$$
\begin{array}{r}
6 \mathrm{EuF}_{3}+8 \mathrm{NH}_{3} \rightarrow 6 \mathrm{EuF}_{2}+6 \mathrm{NH}_{4} \mathrm{~F}(\mathrm{~g})+ \\
\mathrm{N}_{2}(\mathrm{~g}) . \quad \text { (5) }
\end{array}
$$

Jusqu'à $1100^{\circ} \mathrm{C}$ (limite supérieure de température de notre appareillage) l'ammoniac est sans action sur $\mathrm{EuF}_{2}$.

Cas de $\mathrm{TmF}_{3}$. Nous n'avons observé aucune réaction entre $\mathrm{TmF}_{3}$ et $\mathrm{NH}_{3}$ sous pression d'un bar dans l'intervalle de température envisagé $\left(400-1100^{\circ} \mathrm{C}\right)$.

Cas de $\mathrm{CeF}_{4}$. Ce fluorure est réduit par l'ammoniac dès $400^{\circ} \mathrm{C}$ suivant le schéma réactionnel:

$$
\begin{array}{r}
6 \mathrm{CeF}_{4}+8 \mathrm{NH}_{3} \rightarrow 6 \mathrm{CeF}_{3}+6 \mathrm{NH}_{4} \mathrm{~F}(\mathrm{~g})+ \\
\mathrm{N}_{2}(\mathrm{~g}) .
\end{array}
$$

Pour des températures supérieures à $900^{\circ} \mathrm{C}$ la réaction (4) intervient, conduisant à la formation du fluoronitrure de cérium trivalent $\mathrm{CeN}_{x} \mathrm{~F}_{3-3 x}$.

\section{Etude Structurale}

Les fluoronitrures de lanthane, de cérium et de gadolinium cristallisent dans le système cubique de type fluorine. Quel que soit le traitement thermique, nous n'avons jamais observé de déformation de la maille fluorine ni de raies de surstructure témoignant d'un ordre anionique. Le paramètre de $\mathrm{Gd}_{3} \mathrm{NF}_{6}$ est $a=5.617 \pm 0.001 \AA$.

La Fig. 2 montre pour $\mathrm{LaN}_{x} \mathrm{~F}_{3-3 x}$ et $\mathrm{CeN}_{x} \mathrm{~F}_{3-3 x}$ la variation du paramètre en fonction de la composition. Elle correspond it des échantillons "trempés" et confirme pour

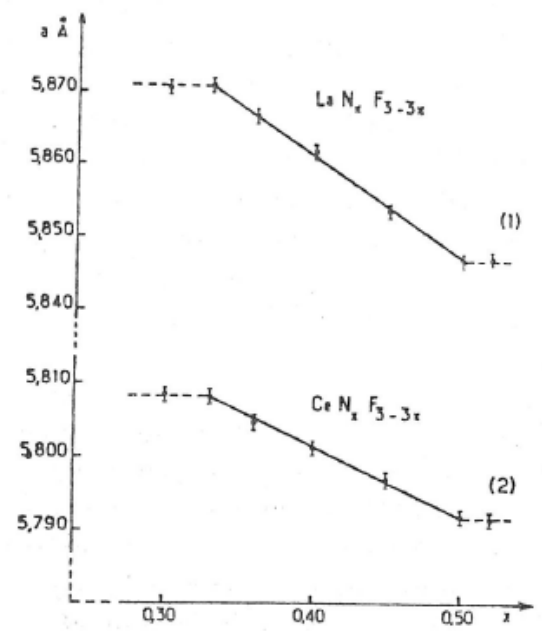

FIG. 2. Variation du paramètre avec $x$ des fluoronitrures de lanthane (1) et de cérium (2).

ce traitement les limites de composition proposées: le rapport (nombre d'anions)/ (nombre de cations) est compris entre 2 et 2.33. On note que le paramètre diminue quand le taux en azote augmente, autrement dit quand le nombre d'anions décroît, la composition se rapproche alors de celle d'une maille fluorine.

Les solutions solides observées pouvaient correspondre théoriquement soit à une insertion d'anions dans les sites vacants d'une maille fluorine stoechiométrique, le nombre de cations par maille élémentaire restant constant et égal à 4 , soit éventuellement au maintien du réseau anionique, le nombre de cations diminuant (Fig. 3).

Dans le premier cas les solutions solides correspondraient ̇̀ la formule structurale: $\operatorname{Ln} X_{2+y}$ où $0<y<0.33$, avec $y=1-2 x$ $(X=\mathrm{N}, \mathrm{F})$.

Dans le second cas à: $L n_{2 / 2+y} X_{2}$.

La Fig. 4 représente pour le lanthane la variation des densités calculées (avec 4 motifs par maille) dans chacune de ces deux hypothèses structurales, ainsi que les valeurs expérimentales observées pour le fluoronitrure de lanthane. Elle montre que l'évolution de la composition correspond sans ambiguité à une insertion dans le réseau fluorine. 


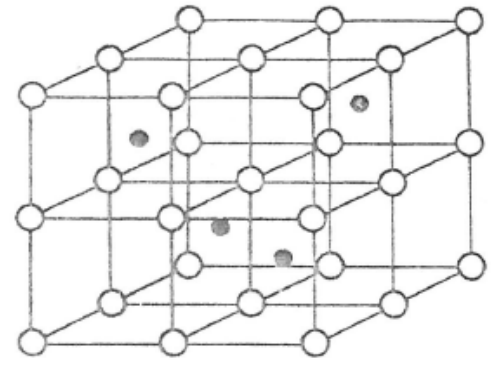

- cation

(a)

anion

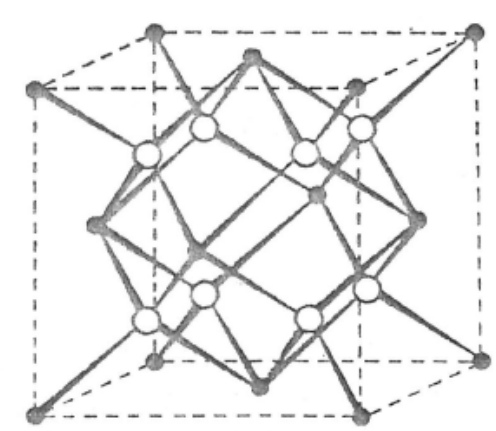

(b)

FIG. 3. Structure de la fluorine. (a) anion en $(0,0,0)$; (b) cation en $(0,0,0)$.

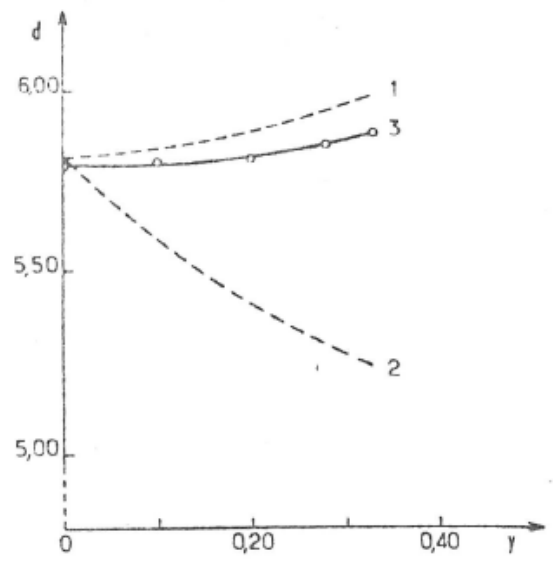

Fig. 4. Variation de la densité en fonction de la composition du fluoronitrure de lanthane 1, s.s. à anions interstitiels; 2 , s.s. déficitaire en cation; 3 , valeurs expérimentales.

Deux modèles étaient susceptibles de rendre compte de cette non-stoechiométrie:

- le premier a été proposé par Bevan lors de l'étude du système YOF-YF $F_{3}$, où ap- paraissent des phases dérivées de la fluorine correspondant à un rapport (nombre d'anions)/(nombre de cations) $>2$ (15). Elles correspondent à l'empilement de blocs fluorine entre lesquels s'insèrent les anions suplémentaires. Mais elles sont caractérisées par l'apparition de raies de surstructure que nous n'avons jamais observées sur les clichés de Guinier même pour des temps de pose de 10 heures. Ce modèle ne semble donc pas valable ici.

-le deuxième modèle a été proposé par Willis lors de l'étude par diffraction neutronique de monocristaux de composition $\mathrm{UO}_{2+x}$ $(0<x<0.25)(16,17)$. Aucune déformation de la maille fluorine n'est observée. Les mesures de densité montrent que, quant $x$ croît, la variation de composition est due à une insertion d'oxygène dans la structure fluorine et non à la crèation de lacunes dans le réseau cationique. Dans le modèle proposé la maille fluorine contient en fait trois types d'oxygène: $\mathrm{O}, \mathrm{O}^{\prime}$ et $\mathrm{O}^{\prime \prime}$ (Fig. 5).

-les atomes $\mathrm{O}$ occupent les sites normaux de la fluorine de coordinence tétraédrique, mais une partie de ces sites deviennent vacants dans la solution solide.

-les atomes $\mathrm{O}^{\prime}$ et $\mathrm{O}^{\prime \prime}$ occupent des positions interstitielles de coordinence octaédrique normalement vacantes dans la maille fluorine (Fig. 3). Mais ces positions ne correspondent pas au centre des octaèdres, clles sont déplacées

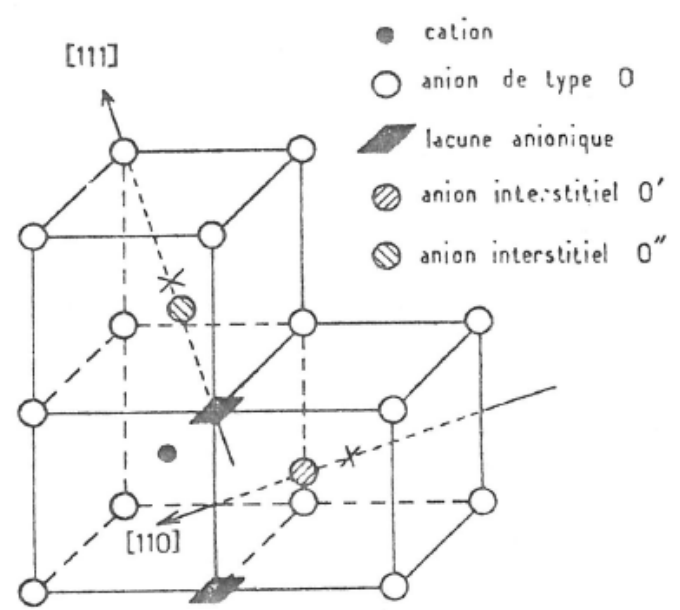

FIG. 5. Positions des anions interstitiels dans le modèle de Willis. 
le long des axes [110] pour $\mathrm{O}^{\prime}$ et [111] pour $\mathrm{O}^{\prime \prime}$ (Fig. 5). Par ailleurs chaque oxygène de type $\mathrm{O}^{\prime}$ est associé à deux lacunes du réseau anionique normal $O$, de sorte qu'il ne possède plus que 6 proches voisins. Les atomes $\mathrm{O}^{\prime \prime}$ par contre sont associés chacun à une seule lacune, de manière que le nombre de proches voisins $\mathrm{O}$ devient égal à 7.

Les oxygènes interstitiels $\mathrm{O}^{\prime}$ et $\mathrm{O}^{\prime \prime}$ apparaissent en nombre variable et s'associent en différentes combinaisons conduisant à la formation de "clusters" en fonction de la composition de la solution solide. La Fig. 6 représente un tel "cluster" constitué par l'association de deux lacunes dans les sites $O$ à deux atomes interstitiels $\mathrm{O}^{\prime}$ et $\mathrm{O}^{\prime \prime}$ (nous l'appellerons le "cluster" $2: 2: 2$ ).

Récemment Cheetham, Fender et Cooper ont utilisé avec succès le modèle de Willis pour rendre compte des données expérimentales fournies par la diffraction de neutrons lors de l'étude du système $\mathrm{CaF}_{2}-\mathrm{YF}_{3}(18)$. Le fait que ce modèle s'applique à deux solutions solides relativement différentes comme $\mathrm{UO}_{2+x}$ et $(\mathrm{Ca}, \mathrm{Y}) \mathrm{F}_{2+x}$ conduit ces auteurs à penser qu'il comporte un caractère

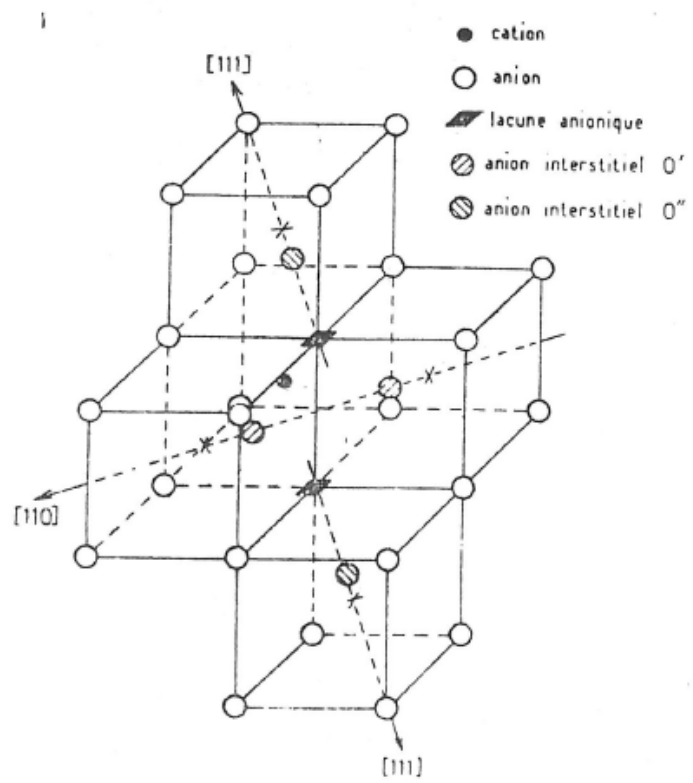

Fili. 6. Cluster 2:2:2. (Les trois chiffres indiquant respectivement le nombre de lacunes $\mathrm{O}$, d'interstitiels $\mathrm{O}^{\prime}$ et $\left.\mathrm{O}^{\prime \prime}.\right)$ général, toutes les fois qu'apparaît une nonstoechiométrie par insertion d'anions dans la structure fluorine (19).

Le modèle de Willis était donc vraisemblablement celui auquel correspondaient les fluoronitrures de lanthanides. Pour vérifier ce point on est conduit à réaliser un calcul d'intensités de diffraction par les plans réticulaires de la structure en affinant les différents paramètres liés à $\mathrm{O}, \mathrm{O}^{\prime}$ et $\mathrm{O}^{\prime \prime}$, de façon à obtenir le meilleur accord avec les intensités mesurées.

Dans le cas de nos fluoronitrures pour lesquels la diffraction $\mathrm{X}$ ne donne que peu de précisions sur la position d'atomes légers comme l'azote et le fluor en présence d'atomes lourds comme ceux des terres rares, la diffraction neutronique est la technique structurale de choix. C'est la méthode que nous envisageons d'utiliser ultérieurement.

Nous avons pensé toutefois qu'un calcul d'intensités de diffraction $X$, réalisé pour des compositions différentes d'une même solution solide, pouvait conduire à des résultats en eux-mêmes très imprécis, mais dont l'évolution pouvait cependant être significative.

Nous avons retenu pour cette étude le fluoronitrure de lanthane qui présente un large domaine d'existence. De plus il est stable à l'air, ce qui facilite les mesures. Les intensités des réflexions ont été déterminées sur poudres par une méthode de comptage statistique. Nous avons retenu les trois compositions $\mathrm{LaN}_{0.45} \mathrm{~F}_{1.05} ; \mathrm{LaN}_{0.40} \mathrm{~F}_{1.80}$ et $\mathrm{LaN}_{0.33} F_{2.00}$. Nous avons affecté à l'azote et au fluor le même facteur de diffusion et nous avons placé les atomes dans les positions proposées par Willis:

$\begin{array}{llll}\text { La: } & 0 & 0 & 0 \\ \mathrm{O}(\mathrm{N} \text { ou F }): & \frac{1}{4} & \frac{1}{4} & \frac{1}{4} \\ \mathrm{O}^{\prime}(\mathrm{N} \text { ou } \mathrm{F}): & \frac{1}{2} & u & u \\ \mathrm{O}^{\prime \prime}(\mathrm{N} \text { ou } \mathrm{F}): & v & v & v\end{array}$

La somme des taux d'occupation des sites anioniques $M_{0}, M_{0}$, et $M_{0}$. était fixée pour chaque échantillon par la composition du fluoronitrure. Chacun de ces taux était obtenu en minimisant le facteur de reliabilité.

Les résultats pour 14 raies sont rassemblés au Tableau II. Les facteurs de reliabilité: 
TABLEAU 1

Résultats de l'Analyse Chimique des Fluoronitrures de Lanthane, de Cérium et de Gadolinium CORRESPONDANT À $x=0.33$

\begin{tabular}{|c|c|c|c|c|c|c|}
\hline & \multicolumn{2}{|c|}{$\mathrm{LaN}_{0.33} \mathrm{~F}_{2}$} & \multicolumn{2}{|c|}{$\mathrm{CeN}_{0.33} \mathrm{~F}_{2}$} & \multicolumn{2}{|c|}{$\mathrm{GdN}_{0.33} \mathrm{~F}_{2}$} \\
\hline & $\%$ théor. & $\%$ exp. & $\%$ théor. & $\%$ exp. & \% théor. & $\%$ exp. \\
\hline$L n=\mathrm{La}, \mathrm{Ce}, \mathrm{Gd}$ & 76.50 & $75.8 \pm 0.8$ & 76.66 & $76.0 \pm 0.8$ & 78.65 & $78.1 \pm 0.8$ \\
\hline $\mathrm{N}$ & 2.57 & $2.55 \pm 0.03$ & 2.55 & $2.51 \pm 0.03$ & 2.33 & $2.28 \pm 0.03$ \\
\hline $\mathrm{F}$ & 20.93 & $20.7 \pm 0.2$ & 20.79 & $20.7 \pm 0.2$ & 19.0 & $19.1 \pm 0.2$ \\
\hline
\end{tabular}

TABLEAU II

Valeurs des Intensités Mesurées fit Calculées de 14 Raies pour Chacune des Compositions Étudiées

\begin{tabular}{|c|c|c|c|c|c|c|}
\hline \multirow[b]{2}{*}{$h k l$} & \multicolumn{2}{|c|}{$\mathrm{LaN}_{0.45} \mathrm{~F}_{1.03}$} & \multicolumn{2}{|c|}{$\operatorname{LaN}_{0.40} F_{1.80}$} & \multicolumn{2}{|c|}{$\mathrm{LaN}_{0.33} \mathrm{~F}_{2}$} \\
\hline & $I_{o}$ & $I_{c}$ & $I_{o}$ & $I_{c}$ & $I_{o}$ & $I_{c}$ \\
\hline 111 & 1000 & 940 & 1000 & 922 & 1000 & 951 \\
\hline 200 & 288 & 287 & 379 & 261 & 338 & 270 \\
\hline 220 & 407 & 539 & 437 & 457 & 357 & 519 \\
\hline 311 & 417 & 437 & 472 & 547 & 372 & 370 \\
\hline 222 & 66 & 79 & 110 & 82 & 101 & 73 \\
\hline 400 & 86 & 75 & 55 & 78 & 49 & 73 \\
\hline 331 & 129 & 162 & 189 & 157 & 220 & 140 \\
\hline 420 & 79 & 100 & 86 & 92 & 108 & 84 \\
\hline 422 & 120 & 144 & 132 & 146 & 146 & 134 \\
\hline $511 / 333$ & 121 & 118 & 98 & 123 & 94 & 105 \\
\hline 440 & 36 & 46 & 54 & 54 & 26 & 43 \\
\hline 531 & 170 & 137 & 113 & 131 & 79 & 123 \\
\hline 600,442 & 96 & 66 & 46 & 58 & 48 & 58 \\
\hline 620 & 135 & 83 & 136 & 85 & 93 & 74 \\
\hline
\end{tabular}

$P_{l}=\Sigma I_{m}, \ldots I_{c} I I_{m}$, voisins de $10 \%$ sont satisfaisants pour un calcul effectué à partir des données d'un spectre de poudre et montrent que le modèle de Willis peut effectivement être retenu pour les fluoronitrures de lanthanides.

Le manque de précision dû à la faible contribution des atomes légers à l'intensité des pics de diffraction par rapport à celle du lanthane entache d'une erreur importante les paramètres positionnels, ainsi que les taux doccupation des sites anioniques. Ces dernieres valeurs étaient d'ailleurs plus incertatines deans le cas de $M_{O^{*}}$ et $M_{O^{*}}$ que pour $M_{\mathrm{O}}$. La valeur absolue de ces paramètres ne peut donc être retenue. Cependant l'évolution des taux d'occupation en fonction de la composition peut paraître significative. Les valeurs sont rassemblées au Tableau III.

On note que $M_{0}$ reste sensiblement constant, alors que $M_{\mathrm{O}}$, et la teneur en azote diminuent simultanément, $M_{\mathrm{O}^{\prime \prime}}$ augmentant avec la quantité de fluor.

Dans le cas des phases $(\mathrm{Ca}, \mathrm{Y}) \mathrm{F}_{2+x}$ Cheetham et al. observent une augmentation simultanée de $M_{\mathrm{O}^{\prime}}$ et $M_{\mathrm{O}^{\prime \prime}}$ avec $x$ (18). On est donc tenté de penser que dans les fluoronitrures l'azote va préférentiellement dans les sites $\mathrm{O}^{\prime}$ et le fluor dans ceux de type $\mathrm{O}^{\prime \prime}$, les sites tétraédriques $O$ étant occupés essentiellement par des atomes de fluor.

La détermination du potentiel de chacun des sites $\mathrm{O}, \mathrm{O}^{\prime}$ et $\mathrm{O}^{\prime \prime}$ permettrait peut-être de mettre en évidence la tendance de l'azote à occuper préférentiellement l'un des sites. Mais l'imprécision sur la détermination des paramètres positionnels de $\mathrm{O}^{\prime}$ et de $\mathrm{O}^{\prime \prime}$ et le caractère covalent de la liaison L.a-N rendait un tel calcul très aléatoire.

\section{TABLEAU III}

Valeurs des Taux d'Occupation $M_{\mathrm{O}}, M_{\mathrm{O}}$ e et $M_{\mathrm{O}^{\prime}}$ " puur Chacune des Compositons Étudiées

\begin{tabular}{lccc} 
& $\mathrm{LaN}_{0.45} \mathrm{~F}_{1.65}$ & $\mathrm{LaN}_{0.40} \mathrm{~F}_{1.80}$ & $\mathrm{LaN}_{0.33} \mathrm{~F}_{2}$ \\
\hline$M_{\mathrm{O}}$ & 1.6 & 1.7 & 1.7 \\
$M_{\mathrm{O}^{\circ}}$ & 0.5 & 0.3 & 0.2 \\
$M_{\mathrm{O}^{*}}$ & 0 & 0.3 & 0.4 \\
\hline
\end{tabular}


Les données cristallochimiques dont nous disposons confirment l'instabilité de l'azote dans les sites tétraédriques $\mathrm{O}$ de la structure fluorine. En effet la phase stoechiométrique qui doit correspondre au maximum d'azote dans ces sites $\mathrm{LaN}_{0.50} \mathrm{~F}_{1.50}$ est instable et ne peut être isolée que par trempe. Après recuit on observe effectivement une démixtion:

$$
\mathrm{LaN}_{0.50} \mathrm{~F}_{1.50} \rightarrow 0.94 \mathrm{LaN}_{0.47} \mathrm{~F}_{1.59}+
$$

$0.06 \mathrm{LaN}$.

Cette instabilité semble encore accentuée dans le cas du gadolinium de taille plus faible et qui donne donc naissance à une maille plus petite, pour lequel seule la phase $\mathrm{GdN}_{0.33} \mathrm{~F}_{2}$ a pu être isolée. Les atomes d'azote, trop gros pour occuper un site $\mathrm{O}$ migrent obigatoirement en $\mathrm{O}^{\prime}$, de coordinence 6 , où ils disposent de davantage de place.

On pouvait se demander à première vue pourquoi parmi les deux sites interstitiels $\mathrm{O}^{\prime}$ et $\mathrm{O}^{\prime \prime}$ susceptibles d'être occupés par l'azote, O' l'était plutôt que O". Dans le cas de O', comme le montrent les figures 5 et 6 , le nombre et la répartition des charges anioniques voisines sont énergétiquement plus favorables, l'azote se déplaçant le long de l'axe [110] vers les lacunes anioniques.

\section{Propriétés Magnétiques de $\mathbf{G d}_{3} \mathrm{NF}_{6}$}

Lat Fig. 7 montre la variation thermique de l'inverse de la susceptibilité massique de $\mathrm{Gd}_{3} \mathrm{NF}_{\text {, }}$ entre 30 et $300 \mathrm{~K}$. Dans le domaine de température étudié elle suit une loi de CurieWeiss $\left(\theta_{p}=-18 \mathrm{~K}\right)$. Le moment magnétique

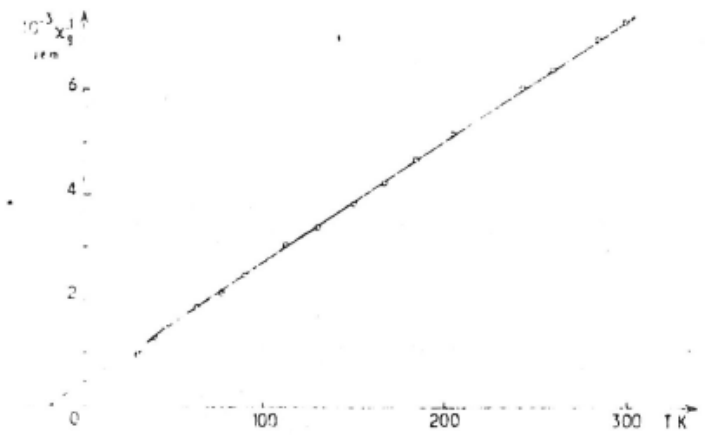

Fici. 7. Variation thermique de la susceptibilité réciproque de $\mathrm{Gd}_{3} \mathrm{NF}_{6}$.

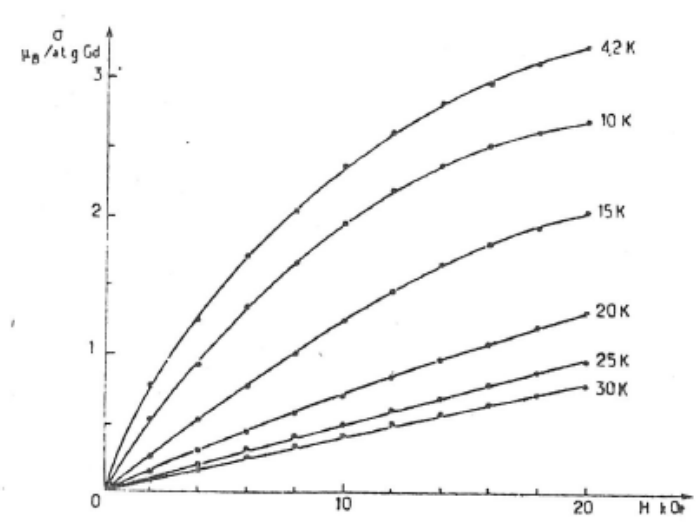

FIG. 8. Variation de l'aimantation en fonction du champ pour $\mathrm{Gd}_{3} \mathrm{NF}_{6}$.

effectif mesuré $\left(8.08 \mu_{\mathrm{B}}\right)$ est en bon accord avec la valeur calculée $\left(7.94 \mu_{\mathrm{B}}\right)$ pour un ion dans l'état fondamental ${ }^{8} S_{7 / 2}$.

En-dessous de $30 \mathrm{~K}$ la variation de l'aimantation en fonction du champ appliqué n'est plus linéaire comme le montre la Fig. 8. De plus le calcul de l'aimantation sporitanée selon la méthode d'extrapolation de Belov (20) et Kouvel (2I) donne une valeur de l'ordre de $0.6 \mu_{\mathrm{B}}$ à $4.2 \mathrm{~K}$. Ces résultats ne semblent pas attribuables à la présence de traces d'impuretés, que l'analyse chimique et radiocristallographique ne permettent pas de déceler. Vu la méthode de préparation, cellesci ne pourraient être que lc gadolinium métallique ou son nitrure, tous deux ferromagnétiques, mais dont les températures de Curie sont respectivement de 289 K (22) et de $69 \mathrm{~K}(23)$. II semble donc que l'aimantation spontanée observée soit une propriété du fluoronitrure lui-même

La valeur approximative de celle-ci $\left(0.6 \mu_{\mathrm{B}}\right)$ représente $8 \%$ environ de celle qui correspondrait à l'alignement de tous les spins $\left(7 \mu_{\mathrm{B}}\right)$. Elle est trop importante pour pouvoir correspondre à un phénomène de "spin canting"; dans ce cas en effet les valeurs déterminées pour l'aimantation spontanée sont de 100 à 1000 fois plus faibles (24).

Dans le réseau du fluoronitrure l'ion $\mathrm{Gd}^{3+}$ est entouré de 12 proches voisins et de 6 seconds voisins gadolinium caractérisés par les intégrales d'échange $J_{1}$ et $J_{2}$. L'étude radiocristallographique a montré que l'azote 
se place essentiellement en coordinence 6 . De plus la distance entre le gadolinium et ses seconds voisins (de l'ordre de $5.62 \AA$ ) est telle que l'on peut exclure des interactions cation-cation. Il est donc tentant de penser à un couplage par superéchange pour un angle voisin de $180^{\circ}$ par l'intermédiaire de l'azote situé en $O^{\prime}$ qui rendrait compte de la valeur négative de $O_{p}$. Le fluor en sites interstitiels conduirait en effet aux mêmes interactions, mais très affaiblies.

On peut donc se demander quel type d'interaction peut être responsable de la composante ferromagnétique. Par référence aux chalcogénures d'europium on pouvait envisager une interaction cation-cation (25). Cependant le mécanisme proposé pour ces chalcogénures fait intervenir les orbitales $5 \mathrm{~d}$ de l'europium. Il semble difficile dans le cas du gadolinium d'envisager un tel mécanisme, on sait en effet que pour ce cation les orbitales $4 \mathrm{f}$ et $5 \mathrm{~d}$ sont séparées par une énergie qui est le double environ de celle observée pour $\mathrm{Eu}^{2+}$. Des interactions de type $R K K Y$ sont également exclues en raison du caractère isolant de $\mathrm{Gd}_{3} \mathrm{NF}_{6}$.

Une interprétation du ferromangettisme relativement important observé pour le fluoronitrure ne pourra être proposée qu'après étude plus précise de la structure cristallographique et détermination de la structure magnétique par diffraction de neutrons.

\section{Discussion et Conclusions}

Ce travail met en évidence l'existence pour le lanthane, le cérium et le gadolinium de no fluoronitrures $L n \mathrm{~N}_{x} \mathrm{~F}_{3-3 x}$.

Dans le cas du lanthane et du cérium ces phases présentent un large domaine d'existence:

$$
\begin{array}{ll}
0.33 \leqslant x \leqslant 0.50 & \begin{array}{l}
\text { pour des échantillons } \\
\text { trempés }
\end{array} \\
0.33 \leqslant x \leqslant 0.47 & \begin{array}{l}
\text { pour des échantillons } \\
\text { recuits }
\end{array}
\end{array}
$$

L'étude radiocristallographique qui fait appel ì un modèle de "cluster" proposé par Willis semble montrer que l'azote occupe préférentiellement les sites octaédriques au sein du réseau fluorine.

Pour le gadolinium de rayon ionique inférieur aux précédents, seule une phase de composition $\mathrm{Gd}_{3} \mathrm{NF}_{6}$ a pu être observée, quel que soit le mode de préparation utilisé. Dans le cas du thulium de taille plus petite encore, nous n'avons pu mettre en évidence de fluoronitrure.

Les résultats obtenus tendent à montrer que la teneur possible en azote des fluoronitrures formés diminue avec la taille de la terre rare. L'azote apparaît comme un élément d'autant plus déstabilisant que celle-ci est plus petite.

Pour chacune des phases observées la limite inférieure de la teneur en azote correspond à $x=0.33$, soit à la formulation $\operatorname{Ln} \mathrm{N}_{0.33} \mathrm{~F}_{2}$. Pour une telle formule la majorité du fluor semble comporter une coordinence tétraédrique, la totalité de l'azote une coordinence octaédrique, distribution pour laquelle la stabilité semble donc maximale.

L'absence d'un fluoronitrure de thulium de structure dérivée de la fluorine pourrait s'expliquer par:

-la déstabilisation accrue de l'azote en site interstitiel, résultat de la diminution du paramètre de la maille fluorine, dont la. valeur obtenue par extrapolation correspondrait à $\mathrm{a}=5.48 \AA$ pour une phase de composition $\mathrm{Tm}_{3} \mathrm{NF}_{\mathrm{t}}$.

---le fait que pour des coordinences $\&$ ou plus élevées les sites correspondants occupés par le thulium sont de basse symétrie, propriété dont s'accomode mal la structure fluorine. Une étude comparative de la coordinence généralement adoptée par les ions terre rare montre en fait que celle-ci diminue du lanthane au lutétium et qu’à coordinence égale la symétrie du site diminue très fortement avec la taille du cation (26-28).

\section{Bibliographie}

1. S. Andersson, Arkit, Kemi 26, 521 (1967).

2. R. JuZA, R. Sievers, ET W. JunG, Naturwissenschaften 53, 551 (1966)

3. R. Juza et R. Sievers, Z. Anorg. Allgem. Chem. 363,258 (1968). 
4. K. Yoshinara, M. Kanno, et T. Mukaïbo, J. Inorg. Nuclear Chem. 31, 985 (1969).

5. S. Andersson, J. Solid State Chem. 1, 306 (1970).

6. P. Ehrlich, W. Linz, et H. J. Steifert, Naturwissenschaften 58, 219 (1971).

7. J. Galy, M. Jaccou, et S. Andersson, C.R. Acad. Sci. 272, 1657 (1971).

8. R. Marchand et J. Lang, Mat. Res. Bull. 6, 845 (1971).

9. W. Jung et R. Juz.A, Z. Anorg. Allgem. Chem. 399, 129 (1973).

10. W. Jung et R. JuzA, Z. Anorg. Allgem. Chem. 399, 148 (1973).

11. B. Tanguy, M. Pezat, J. Portier, et P. HagenMUller, Mat. Res. Bull. 6, 57 (1971).

12. B. Tanguy, M. Pezat, J. Portier, et P. HagenMUller, C.R. Acad. Sci. 274, 1344 (1972).

13. F. Anselin, C.R. Acad. Sci. 256, 2616 (1963).

14. B. TanguY, thèse de Doctorat ès Sciences, Université de Bordeaux I (1972).

15. D. J. M. Bevan, R. S. Cameron, A. W. ManN, G. Braver, et V. Roether, Inorg. Nucl. Chem. Letters 4, 241 (1968),

16. B. T. M. Willis, Proc. Brit. Ceram. Soc. 1, (1964a).
17. B. T. M. Willis, J. Physique 25, 431 (1964).

18. A. K. Cheetham, B. E. F. Fender, et M. J. Cooper, J. Phys. C, Solid State Phys. 4, 3107 (1971).

19. A. K. Cheetham, B. E. F. Fender, D. Steele, R. I. TAYLOR, ET B. T. M. Willis, Solid State Comm. 8, 171 (1970).

20. K. P. Belov et A. N. Goryaga, Fiz. Met. Metall. 2, 3 (1956)

21. J. S. Kouvel, Gen. El. Res. Lab. Report. n57, RL, 1799 (1957).

22. F. Trombe, Ann. Phys. 7, 385 (1937).

23. J. P. Rebouillat et J. J. Weyssie, C.R. Acad. Sci. 259, 4239 (1964).

24. T. MoriYA, in "Magnetism" (G. T. Rado et M. Suhl, Eds.), Vol. 1, p. 87, Academic Press, New York (1966).

25. T. R. MacGuire et M. W. Shafer, J. Appl. Phys. 35, 984 (1964).

26. J. C. Massies, thèse de Doctorat de 3 ème cycle, Université de Bordeaux I (1973).

27. A. Daoudi, thèse de doctorat ès sciences, Université de Bordeaux I (1974).

28. M. Marezio, J. P. Remeika, et P. D. Dernier, Acta Crystallogr. B26, 2008 (1970). 